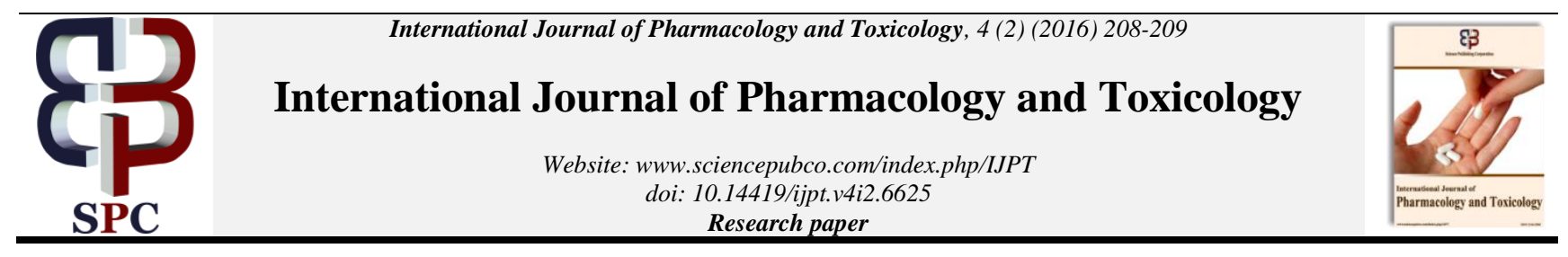

\title{
A qualitative study to analyse adverse drug reaction reporting forms
}

\author{
Thamizhvani.D ${ }^{1}$, Keerthana Brattiya.R ${ }^{1}$, Ramachandra Bhat.C ${ }^{1}$, Stalin.C ${ }^{2} *$ \\ ${ }^{I}$ Department of Pharmacology, Govt Kilpauk Medical College \& Hospital, Chennai-600010 \\ ${ }^{2}$ Pharmacovigilance Associate, Pharmacovigilance Programme of India (PvPI), Indian Pharmacopoeia Commission. Ghaziabad \\ *Corresponding author E-mail: stalinmpharm@gmail.com
}

\begin{abstract}
Introduction: Adverse reactions to drugs cause increase in the hospital admissions. They also cause increased financial burdens to the patients. They can be reduced by increasing the awareness about adverse drug reactions. ADR reporting can create a database help in this regard. To make ADR reporting effective, good ADR reporting form is needed. This study was started to analyse the existing ADR forms of different countries and identify the possible improvements that can be made.

Material and methods: ADR Reporting forms submitted to the Regional Pharmacovigilance Centre were analysed to identify the difficulties faced by the reporters while filling them. ADR reporting forms of different countries were also collected and analysed. Adequacies of these forms were analysed. Based on this qualitative analysis, areas for improvement were identified.

Results: Use of generic names, use of abbreviations and incomplete filling up of the details were observed. Options for causality assessment scales, colouring of mandatory details, categorising ADRs as new or old, dates of intake of concomitant drugs were identified as items to be included in the ADR reporting forms in future.

Conclusion: As per the study's findings and other similar studies, dates of the concomitant drugs, categorisation of ADRs (as known or new ), different colours for the mandatory fields, options for causality assessment scales, whether the ADR is medically confirmed, exact chronology of clinical events are the items which can be included in ADR reporting forms in future. Need for more training for primary reporters in filling up of the ADR reporting form is recognized in this study.
\end{abstract}

Keywords: ADR Reporting Form; Pharmacovigilance; Signals Detection.

\section{Introduction}

Adverse effects to drugs contribute to substantial hospital admissions. They cause increased financial burden to the patients. They also cause increased psychological stress to the patients. One way of reducing these is by creating database of ADRs, identifying ADRs and alerting the physicians about the ADRs. ADR reporting form is designed in different countries based on the local needs of the program for ADR monitoring. One format may be useful for practical aspect of reporting. Another format may be useful for causality assessment while yet another format may be useful for uploading ADRs in Vigiflow. Such variations between countries will make comparison difficult and make causality assessment differ (Bandekar et al. 2010).

With a view to synchronise these needs and develop a new, ideal ADR reporting form, this study was started.

\section{Materials and methods}

ADR reporting forms which were submitted to a Regional Pharmacovigilance Centre were analysed for completeness and comprehensiveness. Forms used in different countries were collected and studied. Requirements to assess the causality as per WHO scale were listed out. Details needed by the Vigiflow software were collected. Analysis of these documents was done to describe the adequacy of these forms. Forms were analysed to find out whether they had adequate information as needed by Vigiflow and WHO scale. Based on these observations, new items were identified for inclusion in any new ADR reporting form.

\section{Results}

\subsection{Analysis of filled - up ADR reporting forms}

Instead of easily understandable words, technical words and abbreviations are used. Instead of generic names, brand names are used .Full details of reporters are not given .Many parts of the ADR forms are left unfilled .Concomitant drugs are given but corresponding details like dates are not given. Clinical history is very brief. Relevant data on lab tests are not given adequately.

\subsection{Adequacy of details needed to perform causality assessment based on WHO scale}

Most of the data needed to do causality assessment based on WHO scale are available in all the ADR reporting forms in this study. Family history may be given more importance in ADR reporting forms in future as ADR s may be familial or may show genetic tendencies.

\subsection{Adequacy of details needed to upload the ADR form} in vigiflow 
Height of the patient is frequently left out.

\subsection{ADR forms were not having provision to answer the following questions}

Is the ADR medically confirmed?

Is the ADR labelled?

\subsection{Essential items which can be included in the new ADR form}

Provision to indicate whether the ADR is already known (new ADR or old ADR) can be included. Mandatory fields can be marked in a separate colour. Options to tick the causality may be included. Dates for the concomitant drugs 'intake may be included.

\section{Discussion}

Different ADR reporting forms were studied. Practical difficulties faced by primary reporters and those doing the causality assessment and those uploading the data were considered. . Areas which need modifications were identified. . Time taken to fill the form is another major area of concern as it differs between different types of health professionals (Rehan et al. 2014). Hence, any new ADR reporting form should be simple and comprehensive.

ADR reporting form must contain all elements necessary to apply WHO scale of causality assessment (Anshi \& Bhatt 2012). Hence, this study attempted to analyse if the ADR reporting forms contain essential elements to apply causality assessment. .If ADR form includes comments on whether the ADR is known or unknown, it will help faster processing and reduce the signal generation time (Hashiguchi et al. 2015). As per this study, it is observed that the forms don't have this entry. In future, ADR forms may mention and highlight the known or new nature of the ADR. This will enable faster processing of new ADR s detected and speed up signal identification process. A clinician who is involved in causality assessment may need the exact chronology of events. If the form gives the exact chronology of events, the assessing person can do it faster and easily (Pia Caduff Janusa Md 2013). It is not to be forgotten that the ADR form has to be user-friendly for online reporting, this aspect too was used while analysing the forms in this study (van Hunsel et al.2012).

Last but not the least; the reporter must know where to send the ADR form (www.who-umc.org/graphics/28521.pdf). As per this study, most of the forms had the address where the forms are supposed to be sent to.

Based on the observations of this study and other studies cited, following recommendations are made:

Training to primary reporters for filling up the ADR reporting form has to be increased.

Any new ADR reporting form in the future may include the items identified in this study.

\section{Conclusion}

As per the study's findings and the similar studies quoted above, dates of the concomitant drugs, categorisation of ADR ( as known or new), different colour for the mandatory fields, ticking facility for the Causality assessment, whether the ADR is medically confirmed, exact chronology of clinical events can be included in ADR reporting forms in future.

\section{Conflict of interest}

\section{Acknowledgement}

The author acknowledges all the staff members of Department Of Pharmacology, Government Kilpauk Medical College and Hospital, Chennai, for helping in this research work.

\section{References}

[1] Bandekar MS, Anwikar SR, Kshirsagar NA (2010) Quality check of spontaneous adverse drug reaction reporting forms of different countries. Pharmacoepidemiol Drug Saf. Nov; 19 (11):1181-5. http://dx.doi.org/10.1002/pds.2004.

[2] Rehan HS, Chopra D, Holani SN, Mishra R (2014) An observational study to compare the contents and quality of information furnished in CDSCO ADR reporting form, yellow card, medwatch and blue form by the healthcare professionals. Int J Risk Saf Med.; 26 (1): $1-8$.

[3] Anshi Singh and Parloop Bhatt (2012) Comparative evaluation of adverse drug reaction reporting forms for introduction of a spontaneous generic ADR form. J Pharmacol Pharmacother. Jul-Sep; 3(3): 228-232.

[4] Hashiguchi M, Imai S, Uehara K, Maruyama J, Shimizu M, Mochizuki (2015) M Factors Affecting the Timing of Signal Detection of Adverse Drug Reactions. PLoS One. Dec 7; 10(12):e0144263. http://dx.doi.org/10.1371/journal.pone.0144263.

[5] Pia Caduff Janusa Md (2013) Medical content needed for analysis.

[6] van Hunsel F, Harmark L, Pal S, Olsson S, van Grootheest K (2012) Drug Saf. Experiences with adverse drug reaction reporting by patients: an 11-country survey. Jan 1; 35(1):45-60.

[7] www.who-umc.org/graphics/28521.pdf 